\title{
Estudio Comparativo de Ansiedad y Disfunción Temporomandibular en Cirujanos Dentistas Residentes de dos Universidades Mexicanas
}

\author{
Comparative Study of Anxiety and Temporomandibular Dysfunctio \\ in Resident Dental Surgeons of Two Mexican Universities
}

\author{
Celia E. Mendiburu-Zavala1; Alfredo Escamilla-Priego²; Pedro Lugo-Ancona ${ }^{1}$ \& María Teresa Zermeño-Loredo³
}

MENDIBURU-ZAVALA, C. E.; ESCAMILLA-PRIEGO, A.; LUGO-ANCONA, P. \& ZERMEÑO-LOREDO, M. T. Estudio comparativo de ansiedad y disfunción temporomandibular en cirujanos dentistas residentes de dos universidades mexicanas. Int. J. Odontostomat., 13(4):458-465, 2019.

RESUMEN: La ansiedad puede ser un detonante de la disfunción temporomandibular (DTM). Se ha mencionado que el trastorno psicológico más frecuente en México es la "ansiedad" con 14,3 \%. El objetivo es correlacionar y comparar los niveles de ansiedad y los grados de DTM en cirujanos dentistas residentes (CDR) de dos universidades mexicanas: la Facultad de Odontología de la Universidad de la Salle Bajío León, Guanajuato (FOULSB), y de la Facultad de Odontología de la Universidad Autónoma (FOUADY) de Yucatán México durante el periodo de septiembre 2017 a febrero 2018. El tipo de estudio fue correlacional, comparativo de cohorte transversal, siendo las variables: ansiedad (niveles según cuestionario de autoevaluación de ansiedad estado/rasgo STAI); signos y síntomas clínicos para el diagnóstico de DTM, (CDI/TTM Grupo I Desórdenes Musculares; Grupo II Desplazamientos discales; Grupo III Artralgia, osteoartritis). El tamaño de la muestra y criterios de inclusión en ambas universidades fueron: CDR en Odontología; que aceptaron participar en el estudio, mediante la firma del consentimiento informado y voluntario; hombres y mujeres; entre 25 a 34 años de edad. Criterios de exclusión: con tratamientos ortodónticos, quirúrgicos, enfermedades sistémicas o neurológicas y que sólo el día que se tomó la muestra presentaron otalgia o cefalea. El tipo de muestreo, por conveniencia. Se utilizó estadística descriptiva e inferencial. Los resultados: 40 CDR de la FOULSB, donde el 92,5\% $(n=37)$ manifestaron tener niveles de ansiedad bajos, y el $7,5 \%(n=3)$ moderado. El 65,8 \% $(n=26)$ presentó diagnóstico de DTM. De los 40 CDR de la FOUADY, 97,5 \% (n=39) declararon tener niveles de ansiedad bajos, y el 2,5\% $(n=1)$ obtuvo un nivel alto. El 67,5\% ( $n=27)$ presentaron signos y/o síntomas de DTM. La conclusión fue que no existieron diferencias estadísticamente significativas, ya que los CDR de ambas universidades presentaron porcentajes de DTM similares y también tuvieron niveles de ansiedad similares.

PALABRAS CLAVE: ansiedad, disfunción temporomandibular, cirujano dentista residente.

\section{INTRODUCCIÓN}

En el ámbito de la salud, no se ha adoptado una única definición sobre el concepto de ansiedad, empleándose sinónimos tales como: angustia, estrés y temor entre otros. Corsini et al. (2012) cita que Bensabat y Selye en su libro sobre "Stress", relacionan ansiedad con angustia y estrés, y establecen la ansiedad como una espera psicológica y temor que se siente ante un acontecimiento, situación difícil o peligrosa.
Álvarez et al. (2012) citan en su artículo que Navas define a la ansiedad como un estado emocional, por lo tanto hay una mezcla de sentimientos, conductas y reacciones o sensaciones fisiológicas. También cita a Papalia, que la define como un estado que se caracteriza por la presencia de sentimientos de aprensión, incertidumbre y tensión que surgen como consecuencia de que el sujeto anticipa una amenaza real o imaginaria (Álvarez et al., 2012). Asimismo, se

\footnotetext{
${ }^{1}$ Facultad de Odontología de la Universidad Autónoma de Yucatán, México.

2 Práctica privada.

${ }^{3}$ Universidad de la Salle Bajío León, Guanajuato, México.
} 
ha de diferenciar lo que es la ansiedad normal, que todos tenemos ante cualquier situación importante estando más activados física y mentalmente y más preparados para responder, de aquella ansiedad que aparece de forma continua y excesiva descontrolando conductas y pensamientos (Álvarez et al., 2012).

El estrés y la ansiedad son dos términos muy afines que guardan relación entre sí, pero que pueden ser diferenciados. Bonilla Silva \& Padilla Infanzón (2015) cita en su artículo que Sierra, Ortega y Zubeidat explican la diferencia entre éstos; el estrés puede ser entendido como la incapacidad del individuo frente a las demandas del ambiente, mientras que la ansiedad es una respuesta emocional ante una amenaza, la cual se manifiesta a nivel cognitivo, fisiológico, motor y emocional. De este modo, el estrés se trata de un proceso adaptativo y de emergencia que permite la supervivencia de la persona. No se considera una emoción en sí misma, sino como un agente generador de emociones. Mientras que la ansiedad alude a un estado de agitación caracterizado por la anticipación del peligro en el que predominan síntomas psíquicos y una sensación de peligro inminente. Añaden estos autores que en la actualidad, "la ansiedad de carácter clínico se caracteriza por presentar una serie de factores entre los que se cuenta que la emoción sea recurrente y persistente, que la respuesta emocional emitida sea desproporcionada en relación a la situación, que sea evocada en ausencia de algún peligro ostensible; y además, que el individuo puede quedar paralizado por un sentimiento de desamparo o se muestre incapaz de realizar conductas adaptativas a la situación con objeto de eliminar dicha ansiedad" (Bonilla Silva \& Padilla Infanzón).

En la clasificación International Statistical Classification of Diseases and Related Health Problems, la ansiedad se puede clasificar como un trastorno neurótico (Álvarez et al., 2011).

En el MILENIO Diario, S.A. de C.V., del 10 de octubre del 2017, mencionó que el trastorno psicológico más frecuente en México es la "ansiedad" con $14.3 \%$ en la población (Milenio, 2017).

En el programa "Salvemos una vida", se abordó el tema "Ansiedad" por el Dr. Salvador González Gutiérrez, presidente de la Red Pro Yucatán, quien mencionó que este trastorno mental es muy frecuente y es una respuesta del organismo desde el punto de vista físico y psicológico, el cual provoca cefaleas, tinnitus, sudoración de manos (hiperhidrosis), sensa- ción de pecho oprimido y dificultades para respirar. Asimismo, aludió que cada persona reacciona diferente ante la misma situación, es decir "cuando un niño desde etapas tempranas comienza a experimentar situaciones de conflictos familiares, va a ir desarrollando una personalidad que llamamos ansiogénica y que se acompaña de mucha inseguridad". Cualquier estimulo negativo, por más pequeño que sea, le provoca incertidumbre. Por otro lado, sugirió que es necesario aprender a manejar las emociones y que esto se logra identificando cada emoción y qué es lo que está creando, como cuadros obsesivos compulsivos y otro tipo de disfunciones (Salazar, 2018).

Entre las alteraciones que pueden ser causadas por la ansiedad esta la disfunción temporomandibular (DTM) que constituye un subgrupo de trastornos musculoesqueléticos relacionados entre sí que implican a la musculatura masticatoria, a la articulación temporomandibular (ATM) y sus estructuras asociadas, y que representa la causa más importante de dolor no dental en la región orofacial. La DTM no sólo es responsable del dolor facial sino que frecuentemente se asocia a dolor craneocervical y cefalea (Mesa Jiménez et al., 2014).

Mediante estudios epidemiológicos ha sido establecido que los signos y síntomas de DTM son comunes en adultos de todas las edades. Los informes han señalado que los signos y síntomas de DTM aumentan con la edad; sin embargo, otros estudios han demostrado una disminución en los síntomas a medida que aumenta la edad. Durante un período de 20 años, las investigaciones sobre DTM han revelado signos y síntomas predominantemente leves ya presentes en la infancia (Majumder et al., 2015).

La DTM es un trastorno de etiología multifactorial en la que intervienen componentes somáticos diversos y psicológicos de origen tensional. En algún momento de la vida de los individuos afectados presentan dolor o incapacidad articular de grado moderado a intenso durante períodos de tiempo variables y aproximadamente $5 \%$ de ellos requiere atención profesional dada la intensidad e incapacidad de la función articular (Velázquez Luna et al., 2013).

En el área de la salud, la educación médica es altamente desafiante y a menudo exige mucho de los recursos mentales de sus estudiantes; aumentando su angustia psicológica y haciéndolos vulnerables a altos niveles de estados afectivos negativos. Comparada con otra educación, la médica se evidencia por 
la alta prevalencia de estrés. Varios estudios han revelado que la incidencia del estrés y las enfermedades relacionadas con éste, como la ansiedad y la depresión en la profesión médica, están aumentando día a día (Shete \& Garkal, 2015).

Los factores psicológicos involucrados en situaciones de DTM pueden dividirse en conductuales, como el bruxismo; emocionales, como el estrés, la ansiedad y la depresión, y cognitiva, con aspectos relacionados con la memoria. Los trastornos de ansiedad son un desafío importante para la sociedad moderna, especialmente en las áreas de atención a los seres humanos, por lo que existe una creciente búsqueda de la comprensión de los factores que pueden provocar el estrés y sus implicaciones (Oliveira Cruz et al., 2015).

Por lo antes expuesto, el objetivo de la presente investigación es correlacionar y comparar los niveles de ansiedad y los grados de DTM en cirujanos dentistas residentes (CDR) de dos universidades mexicanas: la Facultad de Odontología de la Universidad de la Salle Bajío León (FOUSBL), Guanajuato, y de la Facultad de Odontología de la Universidad Autónoma de Yucatán (FOUADY) México durante el periodo de septiembre de 2017 a febrero de 2018 .

\section{MATERIAL Y MÉTODO}

Tipo de estudio: correlacional, comparativo de cohorte transversal. Las variables de estudio fueron: ansiedad (niveles según cuestionario de autoevaluación de ansiedad estado/rasgo STAI (10): 20 a 40= bajo, 41 a $60=$ moderado, 61 a $80=$ alto); signos y síntomas clínicos para el diagnóstico de DTM (dolor miofacial, ruidos articulares, rigidez mandibular, limitación a la apertura bucal, limitación movimientos mandibulares); sexo (hombre, mujer); edad (según la OMS: adultos jóvenes de 25 a 29 años y de 30 a 34); diagnóstico de DTM (según CDI/TTM Grupo I Desórdenes Musculares; Grupo II Desplazamientos discales; Grupo III Artralgia, osteoartritis y osteoartrosis, éste último se excluyó del presente estudio debido a que se investigó a jóvenes aparentemente sanos). El tamaño de la muestra así como los criterios de inclusión en ambas universidades fueron: CDR en Odontología; que aceptaron participar en el estudio, mediante la firma del consentimiento informado y voluntario; hombres y mujeres; entre 25 a 34 años de edad. Criterios de exclusión: con tratamientos ortodónticos, quirúrgicos, enfermedades sistémicas o neurológicas y que sólo el día que se tomó la muestra presentaron otalgia o cefalea. Criterios de eliminación: CDR que decidieron no continuar colaborando durante el estudio, o que no completaron los cuestionarios de autoevaluación de acuerdo a las instrucciones. El tipo de muestreo fue no probabilístico, por conveniencia.

Se aplicó el Cuestionario de Autoevaluación de Ansiedad estado/rasgo (STAI) [Anamnesis Index and the State-Trait Anxiety Inventory (ASTI) de Spielberger, 1988] (Shete \& Garkal). Este cuestionario se compone de dos diferentes escalas preparadas para medir el estado de ansiedad (parte I) y evaluación del rasgo de ansiedad (Parte II). El cuestionario no tuvo tiempo limitado, sin embargo, se utilizó aproximádamente 15 minutos, para la aplicación completa del STAI. Antes de aplicar la autoevaluación, se explicó el procedimiento y se contestaron las dudas. Si durante la autoevaluación, surgió alguna pregunta, el examinador contestó individualmente, de forma que no influyó en el resto de la prueba, ni en los demás examinados. Cada escala tiene 20 ítems (parte I y II) y los participantes anotaron cada respuesta de 0 a 3, obteniendo una puntuación final que podría ser de al menos 20 y en la mayor de 80 o más. Las puntuaciones bajas significaron los bajos niveles de ansiedad y las de alto puntajes, altos niveles de ansiedad. Existe un sistema de corrección con respecto al inventario de la escala de Ansiedad-Estado y de la Ansiedad- Rasgo. Algunos ítems tuvieron que ser recodificados para computar la puntuación total o de alguno de los de Ansiedad E/R: por ejemplo: el ítem 1 "me siento calmado" se puede puntuar $0,1,2,3$. Si bien una puntuación alta en éste no indica ansiedad, sino lo contrario. Por ello, se debió de recodificar la puntuación, siendo la equivalencia: $0=3,1=2,2=1,3=0$. Esto es, si un alumno puntúo con el valor de 0 , en el ítem "me siento calmado" esto equivaldría a un 3 en la recodificación. En la escala Ansiedad Estado se recodificaron los ítems: $1,2,5,8,10,11,15,16,19$, 20. En la de Ansiedad Rasgo se recodificaron los ítems: $21,26,27,30,33,36,39$. Una vez recodificados estos ítems se suman todos los ítems (los 20) de cada escala y se ponderó el nivel de ansiedad de la siguiente manera: 20 a $40=$ bajo nivel de ansiedad; 41 a 60 = moderada nivel de ansiedad; 61 a $80=$ alto nivel de ansiedad.

Por otro lado, para la determinación de DTM, se efectuó un cuestionario al CDR basado en el Índi- 
ce de Criterios Diagnósticos para la Investigación de Trastornos Temporomandibulares (CDI/TTM) de Dworkin \& LeResche (1992), el cual consta de dos ejes: a) El eje 1, se basó en el cuestionario anamnésico y análisis clínico; y b) Eje 2, abarcó el estado psicológico, la discapacidad y el dolor relacionado con los TTM. Este índice presentó criterios estandarizados para la investigación de TTM, maximizó la confiabilidad y minimizó la variabilidad de los métodos de examinación. Solamente se utilizó el eje 1 para los TTM. Los indicadores para el examen de DTM fueron los siguientes: A. Instrucciones generales para el examen: 1 . Todos los aspectos del cuestionario basados en Diagnostic criteria for temporomandibular Disroders (DC/TMD) y el examen clínico fueron completados; 2 . Las mediciones fueron realizadas con los músculos masticatorios en posición de reposo, no recibieron carga o presión adicional en ningún momento; 3 . Los registros en milímetros se anotaron como de dígito único o doble; 4. Los CDR se sentaron en un sillón dental en un ángulo de 90 grados; 5 . El examinador usó guantes, cubre bocas, gorro, lentes y bata clínica desechable según solicita la Norma Oficial Mexicana 013 para el control de infecciones; 6 . Se realizaron los procedimientos de examen ordenada y sistemáticamente como se solicitó en la planilla; 7 . Se efectuaron mediciones clínicas, como el patrón de apertura, apertura mandibular no asistida sin dolor, apertura mandibular máxima asistida, sobremordida vertical, sonidos articulares, clicks en apertura y cierre, derecha e izquierda, movimientos de lateralidad y protrusión, desviación de la línea media, dolor, etc. Todos los datos que se obtuvieron se compilaron en la cédula de investigación. Los datos registrados en las diferentes fases del proyecto fueron organizados en bases de datos, tablas y gráficas.

Se utilizaron pruebas Ji-cuadradas para relacionar variables categóricas, coeficientes de correlación para medir la intensidad de la relación del grado de severidad de la DTM con los niveles de ansiedad, y pruebas parámetricas o no paramétricas para comparar dos o más universidades (CDR de las dos universidades) con respecto a la severidad de la DTM y la ansiedad, ya sean medidas como variables ordinales o como puntaje total obtenido en los cuestionarios.

Las pruebas estadísticas fueron consideradas significativas cuando $P<0,05$ y los paquetes estadísticos que se utilizarán fueron el STATGRAPHICS Centurion XVI v. 16.2.04 (StatPoint, 2013) y el SPSS 21 (IBM SPSS, 2012).

\section{RESULTADOS}

La muestra estuvo formada por $40 \mathrm{CDR}$ de la FOULSB, $57,5 \%(n=23)$ mujeres, y $42,5 \%(n=17)$ hombres. De igual manera, por $40 \mathrm{CDR}$ de la FOUADY, $75 \%(n=30)$ mujeres, y $25 \%(n=10)$ hombres. La edad se distribuyó entre 25 y 34 años.

De los 40 CDR de la FOULSB, el 92,5\% ( $n=37)$ manifestaron tener niveles de ansiedad bajos, y el 7,5 $\%(n=3)$ moderado. De estos mismos, el 65,8 \% $(n=26)$ presentaron diagnóstico de DTM. Los hombres exhibieron el $61,5 \%$ y $38,5 \%$ las mujeres. Los CDR con mayor presencia de DTM, fueron los del rango de 25 a 29 años de edad con un $96 \%(n=25)$. El 76,9\% $(n=20)$ fueron diagnosticados con desplazamiento discal con reducción (grupo II) y el 23,1 \% $(n=6)$ con dolor miofacial (grupo I).

El patrón de apertura con mayor prevalencia, fue el recto con un $77,5 \%(n=31)(55 \%$ en mujeres y el 45 $\%$ en hombres); el de desviación corregida a la derecha ("S"), $15 \%(n=6)$ y el de desviación corregida a la izquierda ("S") 7,5\% (n=3). En 27,5\% ( $n=11)$ se pudo observar que había una desviación de la línea media.

La media de la apertura mandibular máxima no asistida, fue de $51,1 \mathrm{~mm}$ con una desviación estándar (DE) de \pm 2 . Por otro lado, la apertura mandibular máxima asistida fue de $52,7 \mathrm{~mm}$ con una DE de \pm 2 . Cuando se compararon las medidas obtenidas clínicamente, se pudo observar que había una diferencia estadísticamente significativa $(t=3,23, \mathrm{gl}=9$, sig= 0,010 ) en cuanto al click en apertura del lado izquierdo, donde los hombres tenían una media de $3,32 \mathrm{~mm}$ y las mujeres de $2,87 \mathrm{~mm}$.

El 62,5\% $(n=25)$ de los CDR han sentido en algún momento un "click" o "pop" en su articulación cuando abren o cierran su boca o al masticar. En cuanto al sexo, el $68 \%(n=17)$ de las CDR mujeres han sentido en algún momento un "click" o "pop" en su ATM cuando realizan la acción.

Por otro lado, los CDR de la FOUADY el 97,5 \% $(n=39)$ manifestaron tener niveles de ansiedad bajo, y el $2,5 \%(n=1)$ obtuvo un nivel alto de ansiedad.

Por otra parte, el $67,5 \%(n=27)$ de los CDR presentaron signos y/o síntomas de DTM. Con respecto al sexo, el $50 \%(n=20)$ fueron hombres, y el grupo etario con mayor prevalencia fue el de 25 y 29 años 
de edad. En consecuencia, el diagnóstico de DTM con mayor prevalencia entre hombres $(22,2 \%, n=6)$ y mujeres $(44,4 \%, n=12)$ fue el de desplazamiento discal con reducción (grupo II) con $66,7 \%(n=18)$ y $33,4 \%$ $(n=9)$ con dolor miofacial (grupo I).

El patrón de apertura que tuvo mayor prevalencia, fue el recto con un $72,5 \%(n=29) ; 52,5 \%(n=21)$ en mujeres y $20 \%(n=8)$ en hombres, así como en todos los grupos etarios estudiados. En 7 CDR, se observó que había una desviación de la línea media; $28,6 \%(n=2)$ una desviación hacia la derecha, y 71,4 $\%(n=5)$ hacia la izquierda.

La media de la apertura mandibular máxima no asistida, fue de $49,8 \mathrm{~mm}$ con una DE de $\pm 2,1$ y la apertura mandibular máxima asistida fue de 51,7 y una $\mathrm{DE}$ de $\pm 2,2$. No se observaron diferencias estadísticamente significativas entre las medidas obtenidas clínicamente entre hombres y mujeres (t: 1,216, gl: 11,85. Sig: 0,248).

El $72,5 \%(n=29)$ de los CDR han sentido en algún momento un "click" o "pop" en su articulación cuando abren o cierran su boca o al masticar. En cuanto al sexo, el $60 \%(n=24)$ de las mujeres han sentido en algún momento un "click" o "pop" en su articulación cuando abren o cierran su boca o al masticar.
Por último, se buscó determinar si existe una relación entre el nivel de ansiedad y la presencia de DTM, y el tipo de disfunción. Considerando el nivel de medición de las variables (nominales y ordinales), se decidió utilizar el estadístico de Ji Cuadrada.

No existió relación estadísticamente significativa $(\mathrm{c} 2=1,988 ; \mathrm{gl}=1 ; \mathrm{p}=0,338)$ entre ambas variables.

Posteriormente, se analizó si existe relación entre el tipo de DTM y el nivel general de ansiedad. Los resultados señalan que no existió relación, ya que todos los que presentaron DTM tuvieron un nivel de ansiedad leve.

En la Tabla I se comparó la presencia o ausencia de DTM, y el diagnóstico de DTM entre los CDR de ambas universidades y no se observaron diferencias estadísticamente significativas.

Los síntomas reportados en los CDR de ambas universidades se compararon por medio de una prueba de Ji cuadrada: ¿Siente un "click" o un "pop" en su articulación cuando abre o cierra su boca, o al masticar?, ¿Sabe usted si aprieta o rechina los dientes durante el día? y ¿En los últimos 6 meses ha sufrido usted dolores de cabeza o migraña?

Tabla I. Comparación entre presencia y ausencia de DTM, y diagnóstico de DTM en 80 CDR de la Universidad la Salle Bajío y la UADY. 2017-2018

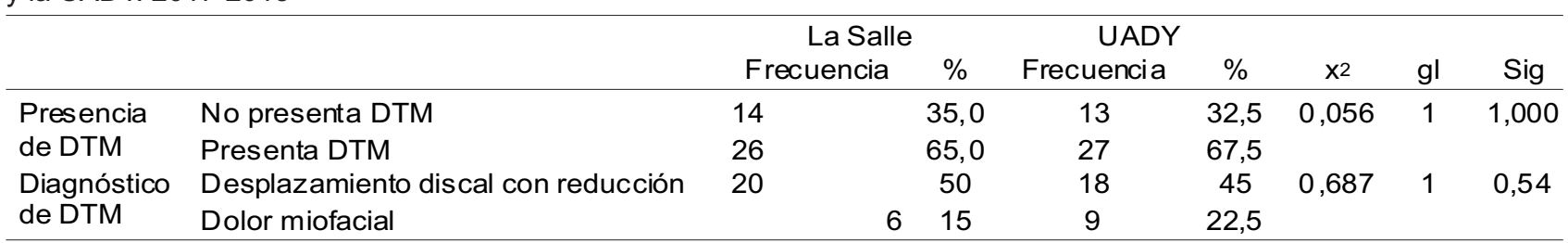

Fuente instrumento de medición $\mathrm{x}^{2}=\mathrm{ji}$ cuadrada

Tabla II. Comparación de los síntomas en los 40 CDR de la Salle Bajío por sexo. 2017-2018.

\begin{tabular}{llllll}
\hline La Salle & Hombres & Mujeres & $X^{2}$ & gl & Sig. \\
\hline Click o Pop al abrir o cerrar la boca & $8(47 \%)$ & $17(73 \%)$ & 3,008 & 1 & 0,107 \\
Rechinar o apretar los dientes & $1(5 \%)$ & $3(13 \%)$ & 0,557 & 1 & 0,624 \\
Dolores de cabeza o mi graña & $5(29 \%)$ & $2(8 \%)$ & 2,906 & 1 & 0,113
\end{tabular}

Fuente: Instrumento de medición $x^{2}$ : ji cuadrada.

Tabla III. Comparación de los síntomas en 40 CDR de la UADY por sexo. 2017-2018.

\begin{tabular}{llllll}
\hline UADY & Hombres & Mujeres & $\mathrm{X}^{2}$ & gl & Sig. \\
\hline Click o Pop al abrir o cerrar la boca & $5(50 \%)$ & $24(80 \%)$ & 3,386 & 1 & 0,103 \\
Rechinar o apretar los dientes & $0(0 \%)$ & $1(3 \%)$ & 0,342 & 1 & 1,000 \\
Dolores de cabeza o mi graña & $0(0 \%)$ & $4(13 \%)$ & 1,481 & 1 & 0,556 \\
\hline Fuente: Instrumento de medión $x^{2}$ ji cuadrada
\end{tabular}

Fuente: Instrumento de medición $x^{2}$ : ji cuadrada. 
No existió diferencias en los síntomas de percepción de "click" o "pop" (c2 = 0,912; gl = 1; $p=0,340)$, rechinar o apretar los dientes $(\mathrm{c} 2=1,920 ; \mathrm{gl}=1 ; \mathrm{p}=$ $0,166)$ y dolores de cabeza o migrañas (c2 $=0,949 ; \mathrm{gl}$ $=1 ; p=0,330$ ).

Tal cómo se observa en la Tabla II, no se encontraron diferencias en ninguno de los síntomas de los CDR de la FOULSB al compararlos por sexo.

De igual forma, no se encontraron diferencias en ninguno de los síntomas en los CDR de la FOUADY cuando se compararon por sexo (Tabla III).

También, se compararon las medidas clínicas entre los CDR de las dos universidades, donde se obtuvieron que hubieron diferencias significativas en la apertura mandibular no asistida sin dolor $(\mathrm{mm})$ y en la apertura mandibular máxima asistida ( $\mathrm{mm}$ ) (TablalV).
Se comparó si existían diferencias estadísticamente significativas entre los CDR de las universidades en la presencia de DTM y los niveles de ansiedad. El primero, se realizó por medio de la prueba Ji cuadrada, y los resultados como se aprecian en la Tabla $V$, señalan que no existió diferencias estadísticamente significativas, ya que los alumnos de ambas universidades presentaron porcentajes de DTM similares (La Salle = $65 \%$; UADY = $67 \%)$.

De igual forma, se compararon los niveles de ansiedad a través de la prueba $U$ de Mann-Whitney. Los resultados señalan que no existió diferencias estadísticamente significativas, pues los CDR de ambas universidades tuvieron niveles similares de ansiedad (FOULSB = 1; FOUADY = 1.05), que corresponden a niveles bajos.

Tabla IV. Comparación de medidas clínicas de 80 CDR de la Universidad la Salle Bajío y la UADY. 2017-2018

\begin{tabular}{|c|c|c|c|c|c|c|c|}
\hline & \multicolumn{2}{|c|}{ La Salle } & \multicolumn{2}{|c|}{ UADY } & \multirow[b]{2}{*}{$\mathrm{T}$} & \multirow[b]{2}{*}{ gl } & \multirow[b]{2}{*}{ Sig } \\
\hline & Media & $\mathrm{DE}$ & Media & DE & & & \\
\hline $\begin{array}{l}\text { Apertura mandibular no asistida } \\
\text { sin dolor }(\mathrm{mm})\end{array}$ & 51,13 & 1,97 & 49,83 & 2,08 & 2,87 & 78 & 0,005 \\
\hline $\begin{array}{l}\text { Apertura mandibular máxima } \\
\text { asistida }(\mathrm{mm})\end{array}$ & 52,71 & 1,98 & 51,68 & 2,20 & 2,18 & 78 & 0,032 \\
\hline Sobremordida Vertical (mm) & 2,60 & 0,85 & 2,76 & 0,57 & $-0,971$ & 78 & 0,335 \\
\hline Clic en apertura derecha $(\mathrm{mm})$ & 3,16 & 0,41 & 3,12 & 0,30 & 0,229 & 23 & 0,768 \\
\hline Clic en apertura izquierda (mm) & 3,07 & 0,32 & 3,08 & 0,36 & $-0,048$ & 19 & 0,962 \\
\hline
\end{tabular}

Fuente: Instrumento de medición T: prueba T de student.

Tabla V. Comparación entre la presencia de DTM y los niveles de ansiedad en 80 CDR de la Universidad la Salle Bajío y la UADY. 2017-2018.

\begin{tabular}{lrrrrrr}
\hline \multicolumn{1}{c}{ Variable } & \multicolumn{2}{c}{ La Salle } & \multicolumn{2}{c}{ UADY } & Estadístico & \multicolumn{1}{c}{ Sig } \\
& Media & DE & Media & DE & & \\
\hline DTM & 0,65 & 0,93 & 0,67 & 0,94 & 0,056 & 1,000 \\
Ansiedad & 1,00 & 0,00 & 1,05 & 0,31 & 780,0 & 1,000 \\
\hline
\end{tabular}

\section{DISCUSIÓN}

En 2011, en Colombia, se identificó el $53 \%$ de la población estudiantil de 21 a 29 años mostraban ansiedad moderada a grado máximo. Fernandes Azevedo et al. (2018) evaluaron a 322 estudiantes de odontología, determinaron que el $65,7 \%$ de éstos, presentaron ansiedad leve, y el 33,3\% moderada. En el presente estudio se encontró una alta prevalencia de ansiedad, correspondiendo al $92,5 \%$ en la FOULSB, de ansiedad leve y $97,5 \%$ en la FOUADY.
En cuanto a DTM, en el presente estudio, se observó que el porcentaje de los CDR de la FOULSB fue similar a los de la FOUADY. González Olivares et al. (2016) estudiaron a 50 residentes de medicina, y pudieron establecer que la prevalencia de DTM en estos fue del $66 \%$, similar a la de ambas universidades mexicanas. Casanova-Rosado et al. (2006) pudieron determinar que el $46,1 \%$ de 506 sujetos de entre 14 y 25 años estudiados en la ciudad de 
Campeche, Campeche, México, presentaron algún grado de DTM. Hedge et al. (2011) comprobaron en su estudio que el $50,5 \%$ de 200 alumnos de odontología estudiados, presentaron síntomas de DTM.

Los resultados obtenidos con relación a la DTM y el sexo, determinaron en el presente estudio que no hubo diferencias significativas entre hombres y mujeres, en ninguna de las dos universidades estudiadas. Smriti et al. (2014) encontraron mayor prevalencia de DTM en mujeres que hombres. Ortiz Cruz et al. (2012) pudieron observar que de los 54 alumnos que estudiaron tenían DTM, el 64,3\% eran mujeres y 35,7 eran hombres.

Con respecto a la clasificación de DTM, en el presente estudio, se pudo observar que la mayor prevalencia en la FOULSB fue el desplazamiento discal con reducción con $76,9 \%$, seguido del dolor miofascial con $23,1 \%$; con resultados similares en la FOUADY, se observó $67 \%$ y $33 \%$ respectivamente. Disímil en resultados al de Wieckiewicz et al. (2014), quienes demostraron que de los 456 estudiantes de diferentes universidades que evaluaron, el $44,3 \%$ tuvo desplazamiento discal con reducción y $18 \%$ dolor miofascial. Manfredini et al. (2012) evaluaron a 520 pacientes que iban en busca de tratamiento de DTM, determinaron que los que tuvieron mayor prevalencia fueron los desórdenes musculares con un $56,4 \%$, seguido de los desplazamientos con un $42 \%$.

MENDIBURU-ZAVALA, C. E.; ESCAMILLA-PRIEGO, A.; LUGO-ANCONA, P. \& ZERMEÑO-LOREDO, M. T. Comparative study of anxiety and temporomandibular dysfunction in resident dental surgeons of two Mexican universities. Int. J. Odontostomat., 13(4):458-465, 2019.

ABSTRACT: Anxiety can be a trigger for temporomandibular dysfunction (TMD). It has been mentioned that the most frequent psychological disorder in Mexico is "anxiety" with $14.3 \%$. The objective is to correlate and compare the levels of anxiety and the degrees of TMD in resident dentists (CDR) of two Mexican universities: the Faculty of Dentistry of the University of La Salle Bajío León, Guanajuato (FOULSB), and the Faculty of Dentistry. Dentistry of the Autonomous University (FOUADY) of Yucatan Mexico during the period from September 2017 to February 2018. The type of study was correlational, comparative crosssectional cohort, the variables being: anxiety (levels according to questionnaire self-assessment of anxiety state / trait STAI); clinical signs and symptoms for the diagnosis of TMD, (CDI I TTM Group I Muscle Disorders, Group II Displacement discs, Group III Arthralgia, osteoarthritis). The size of the sample and inclusion criteria in both universities were CDR in
Dentistry; who agreed to participate in the study, by signing the informed and voluntary consent; men and women; between 25 to 34 years of age. Exclusion criteria: with orthodontic, surgical treatments, systemic or neurological diseases and that only the day the sample was taken showed otalgia or headache. The type of sampling, for convenience. Descriptive and inferential statistics were used. The results: 40 CDR of FOULSB, where $92.5 \%(n=37)$ reported having low anxiety levels, and $7.5 \%(n=3)$ moderate. Sixty-five point eight percent $(n=26)$ presented diagnosis of TMD. Of the 40 CDRs of FOUADY, $97.5 \%(n=39)$ reported having low anxiety levels, and $2.5 \%(n=1)$ had a high level. Sixtyseven point five percent $(n=27)$ presented signs and / or symptoms of TMD. The conclusion was that there were no statistically significant differences, since the CDRs of both universities presented similar DTM percentages and had similar levels of anxiety.

KEY WORDS: anxiety, temporomandibular dysfunction, resident dentist surgeon.

\section{REFERENCIAS BIBLIOGRÁFICAS}

Álvarez, J.; Aguilar, J. M. \& Lorenzo, J. J. La ansiedad ante los exámenes en estudiantes universitarios: relaciones con variables personales y académicas. Electron. J. Res. Educat. Psychol., 10(1):333-54, 2012.

Álvarez, N. S.; Huixtlaca Rojo, C. C.; Espinosa de Santillana, I.; Rebollo Vázquez, J. \& Hernández Jiménez, E. Prevalencia de trastornos temporomandibulares en los alumnos de las clínicas de la Facultad de Estomatología BUAP. Oral, 12(36):66972,2011

Bonilla Silva, K. \& Padilla Infanzón, Y. Estudio piloto de un modelo grupal de meditación de atención plena (mindfulness) de manejo de la ansiedad para estudiantes universitarios en Puerto Rico. Rev. Puertorriq. Psicol., 26(1):72-87, 2015.

Casanova-Rosado, J. F.; Medina-Solís, C. E.; Vallejos-Sánchez, A. A. Casanova-Rosado, A. J.; Hernández-Prado, B. \& AvilaBurgos, L. Prevalence and associated factors for temporomandibular disorders in a group of Mexican adolescents and youth adults. Clin. Oral Investig., 10(1):42-9, 2006.

Corsini, M. G.; Bustos, M. L.; Fuentes, N. J. \& Cantín, M. Anxiety levels in the dental student community. Universidad de La Frontera, Temuco - Chile. Int. J. Odontostomat., 6(1):51-7, 2012.

Dávila Figueras, A.; Ruiz Celis, R.; Moncada Arroyo, L. \& Gallardo Rayo, I. Niveles de ansiedad, depresión y percepción de apoyo social en estudiantes de Odontología de la Universidad de Chile. Rev. Psicol. Univ. Chile, 20(2):147-72, 2011.

Dworkin, S. F. \& LeResche, L. Research diagnostic criteria for temporomandibular disorders: review, criteria, examinations and specifications, critique. J. Craniomandib. Disord., 6(4):301-55, 1992.

Fernandes Azevedo, A. B.; Câmara-Souza, M. B.; Dantas, I. S.; de Resende, C. M. B. M. \& Barbosa, G. A. S. Relationship between anxiety and temporomandibular disorders in dental students. Cranio, 36(5):300-3, 2018.

González Olivares, H.; López Saucedo, F. \& Pérez Nova, A. Prevalencia de disfunción de la articulación temporomandibular en médicos residentes del Hospital de Especialidades Centro Médico Nacional «La Raza». Rev. Odontol. Mex., 20(1):8-12, 2016. 
Hedge, S.; Mahadev, R.; Ganapathy, K. S.; Sujatha, D. \& Patil, B. A. Prevalence of signs and symptoms of temporomandibular disorders in dental students. J. Indian Acad. Oral Med. Radiol., 23(5):316-9, 2011.

Majumder, K.; Sharma, S.; Rao, D.; Siwach, V.; Arya, V. \& Gulia, S. Prevalence and sex distribution of temporomandibular disorder and their association with anxiety and depression in Indian medical university students. Int. J. Clin. Med., 6(8):570-8, 2015.

Manfredini, D.; Arveda, N.; Guarda-Nardini, L.; Segù, M. \& Collesano, V. Distribution of diagnoses in a population of patients with temporomandibular disorders. Oral Surg. Oral Med. Oral Pathol. Oral Radiol., 114(5):e35-41, 2012.

Mesa Jiménez, J.; Torres Cueco, R. \& Fernández de las Peñas, C. Fisioterapia en el tratamiento de la disfunción temporomandibular: una aproximación desde la patología a la guía clínica. Rev. Soc. Esp. Dolor, 21(Supl. 2):14-27, 2014.

Milenio. ¿Cómo andamos de salud mental los mexicanos?. Ciudad de México, Grupo Milenio, 2017. Disponible en: http:// www.milenio.com/salud/salud_mental-mexico-estadisticasdia_mundial-enfermedades-oms-milenionoticias_0_1045695563.html

Oliveira Cruz, A. P.; Rodrigues das Neves, A. F.; Ramires, I. \& Mendonça, M. Effectiveness of mesotherapy on temporomandibular joint disorders. J. Phys. Sci. Appl., 5(4):24954, 2015.

Ortiz Cruz, F.; Martínez Hernández, C. M.; Ríos Gracia, M. C.; Alvarado Salinas, M. C. \& Pérez Montes, G. Factores asociados a la disfunción temporo-mandibular en pacientes de la Facultad de Odontología, Universidad Veracruzana. Odontol. Actual, 9(111):16-22, 2012.

Salazar, J. Vivir Pleno y Sin Ansiedad. Yucatán, Milenio, 2018. Disponible en: https://sipse.com/milenio/salvemos-una-vidasenales-ansiedad-salud-287403.html

Shete, A. N. \& Garkal, K. D. A study of stress, anxiety, and depression among postgraduate medical students. J. Health Res., 2(2):119-23, 2015.

Smriti, J. B.; Patni, V. M.; Mukta, M. \& Gangotri, S. Association between symptoms of temporomandibular disorders and gender, morphological occlusion, and psychological factor in dental students. Int. J. Sci. Study, 2(6):55-8, 2014.

Velázquez Luna, J.; Verdugo Barraza, M. L.; Castro Lara, A. L.; Ramírez Álvarez, M. \& López Zamora, J. H. Disfunción temporomandibular y ansiedad en jóvenes. Rev. Odontol. Latinoam., 5(1):13-7, 2013.

Wieckiewicz, M.; Grychowska, N.; Wojciechowski, K.; Pelc, A.; Augustyniak, M.; Sleboda, A. \& Zietek, M. Prevalence and correlation between TMD based on RDC/TMD diagnoses, oral parafunctions and psychoemotional stress in Polish university students. Biomed Res. Int., 2014:472346, 2014.

\section{Dirección para correspondencia: \\ Dra. Celia E. Mendiburu-Zavala \\ Facultad de Odontología \\ Universidad Autónoma de Yucatán \\ MÉXICO}

\author{
Email: cel_mendi@hotmail.com \\ mzavala@correo.uady.mx
}

Recibido : 02-05-2019

Aceptado: 01-07-2019 